\title{
Coordinación de procesos cognitivos en la resolución de problemas: relación entre geometría y álgebra
}

\author{
Germán Torregrosa Gironés, Universidad de Alicante (España)
}

Recibido el 18 de enero de 2017; aceptado el 28 de julio de 2017

\begin{abstract}
Coordinación de procesos cognitivos en la resolución de problemas: relación entre geometría y álgebra

Resumen

Se presenta una ampliación del modelo de razonamiento configural para el análisis de la resolución de problemas empíricos de geometría, en los que los datos iniciales son numéricos o literales. La extensión del modelo de Razonamiento Configural consiste en la ampliación de significados de las Aprehensiones Operativas y Discursivas (Duval, 1998) y la aceptación del uso del registro algebraico en el discurso generado durante la resolución de problemas de geometría con lápiz y papel. La inclusión del registro algebraico en el modelo se fundamenta en los conceptos de conversión y tratamiento de la Teoría de los Sistemas Semióticos de Duval. Analizamos varias resoluciones a un problema empírico con el nuevo modelo de Razonamiento Configural extendido para evidenciar su potencial.
\end{abstract}

Palabras clave. Sistemas de representación; Razonamiento configural; Problema empírico; Geometría y Álgebra. álgebra

Coordenação de processos cognitivos em solucionando problemas: relação entre geometria e

\section{Resumo}

Este trabalho apresenta uma extensão do modelo de raciocínio configural para a análise dos problemas empíricos de resolução de geometria, no qual os dados iniciais são numérica ou literal. A extensão do modelo de raciocínio Configural consiste no alargamento dos significados deles apreensões operacionais e discursivas (Duval, 1998) e a aceitação do uso do registro algébrico no discurso gerado durante a resolução de problemas de geometria com lápis e papel. A inclusão do registro algébrico no modelo baseia-se os conceitos de conversão e tratamento da Teoria dos Sistemas Semióticos de Duval. Analisamos várias resoluções de um problema empírico com o novo modelo de raciocínio Configural estendido para revelar o seu potencial.

Palavras chave. Sistemas de representação; Raciocínio configural; Problema empírico; Geometria e Álgebra. algebra

Coordination of cognitive processes in problem solving: relationship between geometry and

Para citar: Torregrosa Gironés, G. (2017). Coordinación de procesos cognitivos en la resolución de problemas: relación entre geometría y álgebra. Avances de Investigación en Educación Matemática, 12, $1-17$.

(C) Sociedad Española de Investigación en Educación Matemática (SEIEM). www.seiem.es 


\begin{abstract}
This paper presents an extension of the model of configural reasoning for the analysis of the resolution of empirical problems of geometry, in which the initial data are numerical or literal. The extension of the configural reasoning model consists in the widening of operative and discursive apprehension meanings (Duval, 1998) and the acceptance of the use of the algebraic register in the discourse generated during the resolution of geometry problems with pencil and paper. The inclusion of the algebraic register in the model draws on the concepts of conversion and treatment of the Theory of Semiotic Systems by Duval. We analyzed several resolutions to an empirical problem with the new extended configural reasoning model to show its potential.
\end{abstract}

Key words. Representation systems; Configural reasoning; Empirical problem; Geometry and Algebra.

\title{
Coordination des processus cognitifs dans la résolution des problèmes: relation entre la géométrie et l'algèbre
}

\section{Résumé}

Cet article présente une extension du modèle de raisonnement configural pour l'analyse de la résolution des problèmes empiriques de la géométrie, dans lequel les données initiales sont numériques ou littérales. L'extension du modèle de raisonnement Configural consiste en l'élargissement des significations de ces appréhensions opérationnelles et discursives (Duval, 1998) et l'acceptation de l'utilisation de l'enregistrement algébrique dans le discours généré lors de la résolution des problèmes de géométrie empirique avec crayon et du papier. L'inscription de l'enregistrement du modèle algébrique est basée sur les concepts de la conversion et le traitement de la Théorie des Systèmes Sémiotiques de Duval. Nous analysons plusieurs résolutions à un problème avec le nouveau modèle de raisonnement Configural étendu afin de démontrer son potentiel.

Paroles clés. Systèmes de représentation; Raisonnement configural; Problème empirique; Géométrie et Algèbre.

\section{Introducción}

En este trabajo presentamos una ampliación del modelo de razonamiento configural (Torregrosa \& Quesada, 2007; Prior \& Torregrosa, 2013) al ámbito del análisis de la resolución de problemas de probar de geometría que contienen datos numéricos en el enunciado. En este trabajo denominamos problemas de geometría empíricos a problemas de probar en el que su enunciado asigna cantidades y relaciones entre las cantidades a los objetos geométricos. El uso del modelo extendido en este tipo de problemas permite mostrar que los estudiantes siguen los mismos procesos cognitivos que hemos identificado en las resoluciones de problemas clásicos de probar en Geometría, usando los modos de representación algebraico y geométrico.

El uso del modelo de razonamiento configural, para analizar la resolución de problemas de geometría empíricos, ofrece una aproximación cognitiva y matemática a la comprensión del comportamiento del alumno al resolver este tipo de problemas (Zazkis \& Dubinsky, 1996). La literatura sobre la demostración matemática (prueba) tiene distintos focos: aspectos epistemológicos (Balacheff, 2008; Hanna \& Jahnke, 1996); dificultades del alumno (Harel \& Sowder, 1998); coordinación de estrategias visuales y analíticas (Zazkis, Dubinsky \& Dautermann, 1996); relación entre discurso y demostración (Duval, 1995; Robotti, 2012), relación entre conjeturar y probar (Fiallo \& Gutiérrez, 2017); y enseñanza/aprendizaje de la prueba en varios contextos educativos (Hilbert, Renkl, Kessler \& Reiss, 2008; Ibañes, 2002; Ibañes \& Ortega, 
2005; Komatsu, 2016; Miyazaki, Fujita \& Jones, 2017; Reiss, Heinze, Renkl \& Groß, 2008).

La propuesta integra diferentes aspectos para analizar las dimensiones, epistemológica, cognitiva y discursiva, de la demostración matemática como manera de analizar las aproximaciones analíticas y geométricas de alumnos en la resolución de los problemas empíricos. A partir de la teoría de la representación de Duval y sus estudios sobre los procesos cognitivos de visualización y razonamiento, el modelo del razonamiento configural permite analizar producciones de alumnos cuando resuelven problemas de probar de Geometría, en un entorno de lápiz y papel (Torregrosa \& Quesada, 2007; Torregrosa, Quesada \& Penalva, 2010; Prior \& Torregrosa, 2013). En el uso inicial del modelo de razonamiento configural se consideraron problemas de probar clásicos de geometría con lápiz y papel. Sin embargo, en la etapa escolar y en la Universidad se consideran problemas de contexto geométrico que involucran medidas como datos (problemas de aplicación de resultados geométricos a la vida real). Estos problemas no exigen una demostración matemática formal en sentido estricto, pero sí precisan del conocimiento y uso de las mismas propiedades y resultados geométricos teóricos para resolver los problemas de probar. El análisis de los procesos de resolución de estos problemas empíricos conlleva problemas teóricos que deben abordarse y resolverse con carácter previo. Por ejemplo, no se dan hipótesis consistentes en propiedades geométricas genéricas (dado el triángulo isósceles de la figura) sino datos numéricos o literales (en el triángulo de la figura los lados miden a, a y b cm). Así, las aprehensiones discursivas, las asociaciones de configuraciones puntuales a afirmaciones matemáticas, no siguen el mismo proceso. Además, durante la resolución de problemas empíricos, aparece el registro algebraico para convertir el enunciado dado y los datos en expresiones algebraicas que no se contempla en el modelo de razonamiento configural.

\section{Planteamiento del problema}

Veamos el análisis de la solución a un problema clásico de geometría de probar, utilizando el modelo del razonamiento configural, para lo que necesitamos definir los elementos teóricos que utilizamos.

\subsection{Elementos teóricos en el modelo de razonamiento configural}

Llamamos Razonamiento Configural (RC en adelante) al desarrollo de la acción coordinada entre Aprehensiones Operativas y Aprehensiones Discursivas, realizada por el alumno, asociando afirmaciones matemáticas y/o realizando modificaciones en la configuración inicial, cuando resuelve un problema de geometría mediante lápiz y papel (Torregrosa \& Quesada, 2007; Torregrosa et al., 2010; Prior \& Torregrosa, 2013).

Un alumno realiza una Aprehensión Operativa (en adelante AO) cuando modifica la configuración inicial del problema (Duval, 1995, 1998, 1999). El significado de esta modificación incluye: añadir o quitar elementos geométricos a/de la configuración inicial; reconfigurar las partes (subconfiguraciones) de la configuración inicial, moviendo las subconfiguraciones como piezas de un rompecabezas; identificar un elemento geométrico elemental (segmento, ángulo, triángulo, círculo...) en cuanto que se "aísla" prescindiendo (quitándolo) del resto de la configuración inicial. La modificación se puede realizar tanto física como mentalmente ya que no se representan todas las modificaciones del resolutor. 
Un alumno realiza una Aprehensión Discursiva (en adelante AD) cuando asocia una configuración identificada con una afirmación matemática, para inferir conocimiento que le ayude a resolver el problema (Duval, 1995, 1998, 1999). Esta asociación puede tener dos desencadenantes que corresponden a: la subconfiguración identificada por el alumno le recuerda alguna afirmación matemática, en cuyo caso decimos que hay un cambio de anclaje de visual a discursivo, o bien los conocimientos teóricos del alumno le llevan a buscar la subconfiguración adecuada en la configuración inicial, en cuyo caso decimos que hay un cambio de anclaje de discursivo a visual. El término afirmación matemática incluye teoremas, axiomas, definiciones, propiedades...

$\mathrm{Al}$ analizar las acciones coordinadas $\mathrm{AO} / \mathrm{AD}$, que realiza el alumno para resolver el problema, se clasifica su respuesta según los siguientes desenlaces del RC:

Si hay solución al problema:

- Truncamiento, desenlace que ocurre cuando la coordinación realizada por el alumno proporciona la "idea" que resuelve el problema, permitiéndole generar un proceso deductivo que resuelve el problema. El truncamiento hace referencia al momento en que el alumno "se da cuenta" de la solución. En ese momento finaliza el proceso de razonamiento, entendido como deducir información nueva a partir de otra dada o conocida, y empieza el proceso de generar el discurso deductivo para comunicar la solución. Por tanto, el término no se refiere a ninguna interrupción.

- Conjetura sin demostración, el razonamiento permite generar una solución al problema, pero basada en conjeturas no probadas, como inferencias realizadas en base a percepciones, erróneas o no, de la configuración inicial.

Si no hay solución al problema

- Bucle, que se da cuando se establecen afirmaciones matemáticas que no permiten el avance hacia la solución, de forma que los resolutores vuelven a la situación inicial, una o varias veces, ante la imposibilidad de avanzar en la resolución. Por tanto, decimos que RC desemboca en bucle, cuando se da una situación de bloqueo que no permite el avance hacia la solución.

\subsection{Problemas de geometría clásicos y empíricos}

En los problemas de geometría clásicos de probar intervienen conceptos geométricos: "dos cuerdas paralelas", "recta perpendicular a las cuerdas" que "pasa por el centro de la circunferencia" o "contiene los puntos medios". Se dan situaciones o propiedades geométricas elementales y se pide "Probar que". En los problemas empíricos se dan las medidas de los elementos geométricos y se pide "Calcular". En el problema empírico de la Figura 1, se dan las medidas de las cuerdas y la distancia entre ellas, concretando las opciones de intervención del alumno para resolver el problema. En ocasiones esta concreción es una limitación que no deja lugar a la creatividad, pero en otras ocasiones orienta las acciones para resolver el problema. Igualmente, existen diferencias en la pregunta que se solicita en cada tipo de problema. En los enunciados clásicos a menudo se pide demostrar una propiedad de carácter general: "contiene los puntos medios de ambas cuerdas", mientras que en el problema empírico se pide calcular la medida del radio de la circunferencia, que es un dato concreto de la situación particular propuesta. 


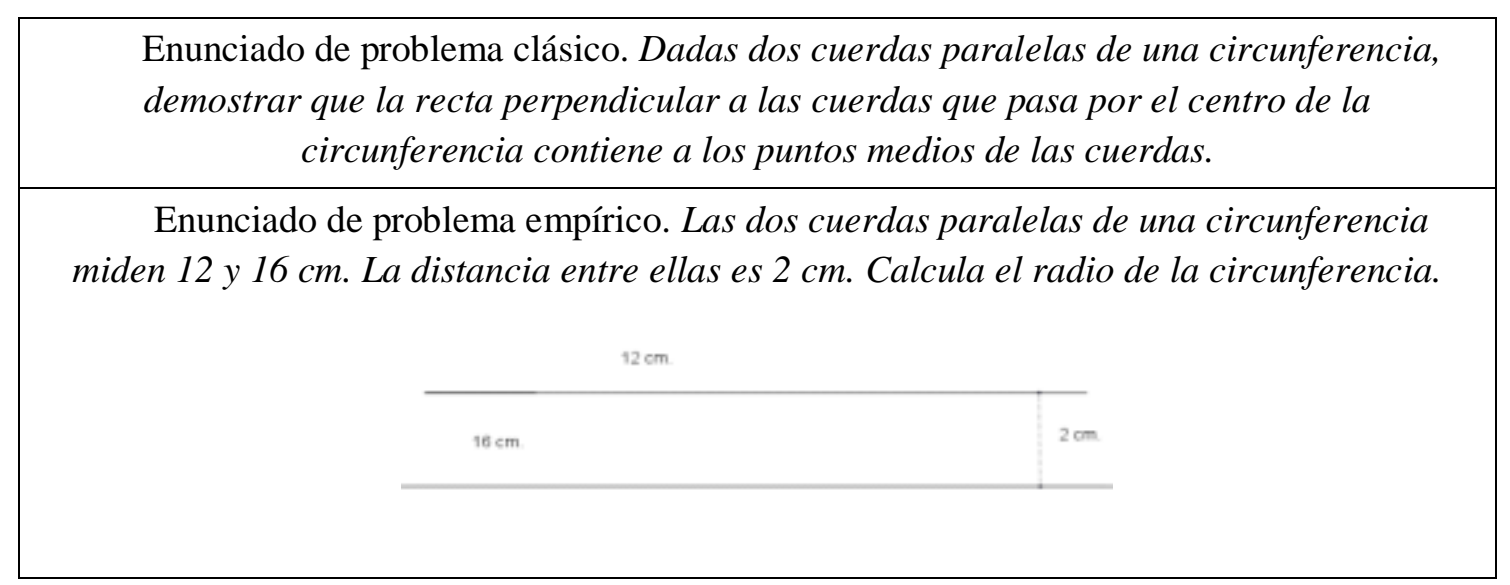

Figura 1. Ejemplos de enunciados de problemas clásico y empírico

El uso de problemas empíricos en las etapas educativas es actualmente predominante en el estudio de la Geometría. Esta tendencia se justificaría entre otras razones por la motivación que aporta este tipo de problemas geométricos; el uso que hacen de ellos los libros de texto en la educación Secundaria; la formación recibida por los graduados de carreras técnicas (ingenieros y arquitectos) en los primeros cursos.

\subsection{Sobre los procesos de resolución}

En cuanto al proceso de resolución, la resolución de los problemas clásicos se apoya en la capacidad de coordinar conocimiento geométrico, para realizar conjeturas que desemboquen en la solución del problema (demostración matemática). Mientras que en los problemas empíricos, el proceso de resolución no conlleva la resolución de cuestiones generales, sino que se estudia una situación geométrica específica y se calcula un dato concreto de ella. Por otra parte, el registro geométrico para comunicar una demostración, de un problema clásico de probar en Geometría, no contempla el uso del registro algebraico (ecuaciones/sistemas de ecuaciones), mientras que la solución a los problemas empíricos se apoya en la posibilidad de usar este registro. Esta situación nos ha llevado a intentar avanzar en la comprensión de la coordinación de los procesos cognitivos de los alumnos (realizando $\mathrm{AO}$ y $\mathrm{AD}$ ) en la resolución de problemas empíricos.

\subsection{Ejemplo de análisis de la resolución de un problema clásico de geometría de probar según el modelo $\mathrm{RC}$}

Dado el problema clásico de geometría de probar que aparece en la Figura 1, analizaremos una resolución según RC:

Problema 1. Dadas dos cuerdas paralelas de una circunferencia, demostrar que la recta perpendicular a las cuerdas que pasa por el centro de la circunferencia contiene a los puntos medios de las cuerdas.

Solución. Realizamos la construcción de una circunferencia de centro $O$ y representamos dos cuerdas paralelas $A B$ y $C D$ (Figura 2). Trazamos los segmentos $O A$ y $O B$ y la recta perpendicular a $A B$ que pasa por $O$, llamando $P$ al punto de intersección. 

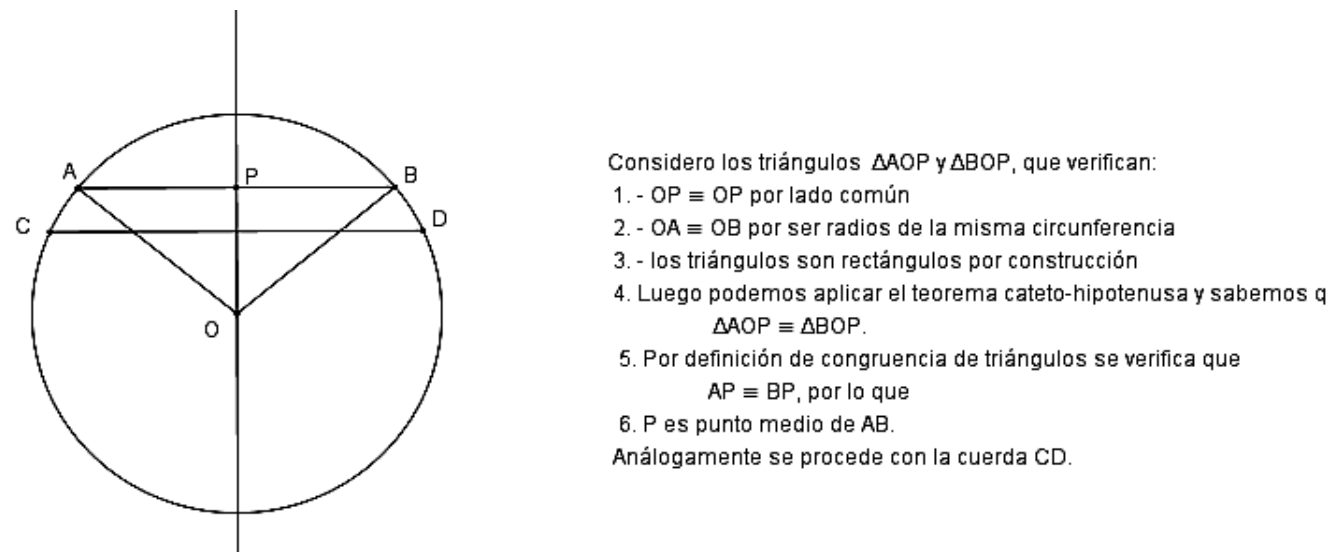

Figura 2. Una solución al problema 1 clásico de geometría de probar

\section{Análisis de la solución desde el modelo RC}

En primer lugar, se representa la situación geométrica descrita en el enunciado mediante la construcción de una circunferencia de centro $O$. Esta acción supone una $\mathrm{AD}$ (recordar la definición de circunferencia) y asociarle la configuración de puntos del plano que equidistan de un punto que llamamos centro (lo que supone una AO).

Del mismo modo, se realiza una AD con la definición de cuerda. Cuando esta se asocia con el segmento $A B$ o el $C D$ identificados, estamos ante una AO. A continuación se traza la recta perpendicular a la cuerda $A B$ que pasa por $O$, lo que constituye una $\mathrm{AO}$ pues añadimos un elemento geométrico a la configuración inicial de circunferencia y cuerdas, cuya definición constituye una $\mathrm{AD}$. Análogamente ocurre con los radios, $\mathrm{AD}$ (definición de radio de una circunferencia) que asociamos a los segmentos identificados $O A$ u $O B$, que trazamos realizando una AO. Llamamos $P$ al punto de corte de $A B$ con la recta $O P$. Todas estas acciones, salvo el trazado de los radios $O A$ y $O B$, corresponden a la realización de una representación de la situación geométrica descrita por el enunciado.

Seguidamente se identifican los dos triángulos $\triangle A O P$ y $\triangle B O P$, mediante una $A O$, ya que se prescinde del resto de la configuración, y se asocia con el criterio de congruencia cateto-hipotenusa mediante una $\mathrm{AD}$. Las condiciones a verificar para aplicar el criterio cateto-hipotenusa corresponden a 1,2 y 3 de la respuesta escrita. En cada condición se observa una AO (identificación de una subconfiguración) asociada a una propiedad/afirmación matemática que constituye la AD.

En 4 se establece la conclusión de que los triángulos rectángulos son congruentes. Por definición de triángulos congruentes (AD) identificamos como congruentes a los segmentos correspondientes $A P$ y $B P(\mathrm{AO})$. De lo que se deduce que $P$ es punto medio de la cuerda $A B$. Análogamente se procedería con el punto medio de la cuerda $C D$. Esta es una respuesta que un experto podría dar al problema de probar planteado y que ha sido usada para describir el análisis de una resolución desde el modelo RC. En esta resolución, el truncamiento se produce cuando el resolutor traza los radios $O A$ y $O B$, ya que en ese momento sabe la solución que busca.

\subsection{Problemática del análisis de la respuesta a un problema empírico según el modelo RC}

Dado el problema empírico de la Figura 1, presentamos el análisis de una solución según RC. 
Problema 2. Las dos cuerdas paralelas de una circunferencia de la figura miden 12 y $16 \mathrm{~cm}$. La distancia entre ellas es de $2 \mathrm{~cm}$. Calcula el radio de la circunferencia.

Solución. Trazamos la circunferencia de centro $O$ y las dos cuerdas $A B$ de $12 \mathrm{~cm}$ de longitud y la $C D$ de $16 \mathrm{~cm}$. La distancia entre ellas es de $2 \mathrm{~cm}$. Construyo el segmento $O P$ perpendicular a $A B$ en $P$ (punto medio de la cuerda $A B$, por lo que sabemos que pasará por el centro de la circunferencia) y trazo los radios $O B$ y $O D$. Llamo $Q$ al punto de intersección de $O P$ con la cuerda $C D$ ( $Q$ también es punto medio de $C D$, por ser $O P$ una perpendicular a la cuerda que pasa por el centro de la circunferencia).
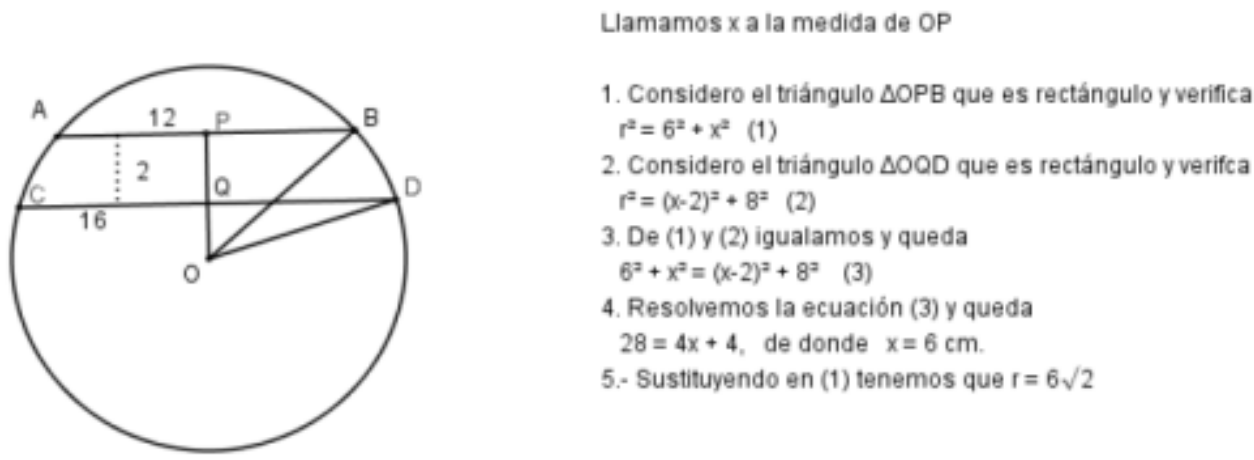

Figura 3. Solución al problema 2 empírico

\section{Análisis de la solución al problema 2 desde el modelo $R C$}

$\mathrm{Al}$ igual que en el problema 1, comenzamos representando la situación geométrica del enunciado mediante la construcción de una circunferencia de centro $O$. Esta acción supone una AD (recordar definición de circunferencia) y asociarle la configuración de puntos del plano que equidistan de un punto que llamamos centro (lo que constituye una AO). Del mismo modo, se realiza una AD con la definición de cuerda y cuando se asocia esta con el segmento $A B$ o el $C D$ identificados, estamos realizando una AO. A continuación se traza la recta perpendicular a la cuerda $A B$ que pasa por $O$, lo que constituye una $\mathrm{AO}$, pues añadimos un elemento geométrico a la configuración inicial de circunferencia y cuerdas, y asociamos la definición, lo que constituye una AD. Análogamente ocurre con los radios, $\mathrm{AD}$ (definición de radio de circunferencia) que asociamos a los segmentos $O A$ y $O B$ y trazamos realizando una AO. Llamamos $P$ al punto de corte de $A B$ con la perpendicular que pasa por $O$, y $Q$ al punto de corte con la cuerda $C D$, lo que equivale a aislar, en cada caso, un punto (realizando una $A O$ ) al que asociamos la propiedad de ser punto medio de sus respectivas cuerdas (realizamos una $\mathrm{AD}$ al vincular la subconfiguración con el conocimiento geométrico "la recta perpendicular en el punto medio de una cuerda pasa por el centro de la circunferencia"). Igualmente, trazamos los segmentos $O B$ y $O D(\mathrm{AO})$ y los asociamos a la definición de radio $(\mathrm{AD})$.

Seguidamente, identificamos el segmento $O P$ (realizando una AO) y le asignamos una medida que llamamos $x$. Esta acción no tiene significado teórico en el modelo RC puesto que no asociamos el segmento con ninguna propiedad/definición/teorema y, por tanto, no realizamos una $\mathrm{AD}$, en el sentido definido en el modelo. Esto mismo ocurre cuando, en la representación geométrica particular del problema, hemos afirmado que $P$ y $Q$ distan, respectivamente de $B$ y de $D, 6$ y $8 \mathrm{~cm}$. También identificamos puntos (AO) pero no asociamos con propiedad/definición/teorema, sino con una medida.

Además, en 1 y 2 en el proceso de resolución (Figura 3) consideramos los triángulos $\triangle O P B$ y $\triangle O Q D$, realizando en cada caso una $\mathrm{AO}$, y les asociamos la propiedad de ser rectángulos mediante una $\mathrm{AD}$, pero aparecen dos ecuaciones cuyo significado no se 
contempla en el modelo RC; esto es, aparece el registro algebraico. En 3, 4 y 5, realizamos unas manipulaciones simbólicas de las ecuaciones que, teniendo sentido en el registro algebraico (tratamiento) puesto que estamos resolviendo el sistema de dos ecuaciones con dos incógnitas, carece de interpretación dentro del modelo RC.

El modelo RC (Torregrosa \& Quesada, 2007; Torregrosa et al., 2010), para analizar las respuestas a los problemas clásicos de probar en geometría, no permite analizar completamente los procesos de resolución en los problemas empíricos. En el enunciado no se dan hipótesis consistentes en propiedades geométricas (dadas dos cuerdas paralelas de una circunferencia), sino datos numéricos o literales concretos (las dos cuerdas paralelas miden 12 y $16 \mathrm{~cm}$ ). Esto implica que las AD (asociaciones de configuraciones puntuales a afirmaciones matemáticas) que el modelo exige identificar no siguen el mismo proceso. Por otra parte, durante la resolución de los problemas empíricos, aparece el registro algebraico para convertir enunciado y datos en expresiones algebraicas. El tratamiento necesario para la solución no se contempla en el modelo RC inicial.

\section{Extensión del modelo RC}

La extensión del modelo RC al análisis de los problemas empíricos consiste en considerar que en las $\mathrm{AD}$, dentro del significado de afirmación matemática que incluía a teoremas, axiomas, definiciones, propiedades,... también se incluyan las condiciones iniciales (hipótesis) de los problemas empíricos, expresados como datos numéricos o literales $(7 \mathrm{~cm} ; x \mathrm{~cm})$. Además, tenemos en cuenta el registro en el que se representa el discurso generado durante la resolución del problema. Debemos considerar, junto al registro geométrico, el registro algebraico dentro de un contexto geométrico. Esta inclusión del registro algebraico en el modelo se justifica, desde el punto de vista cognitivo, mediante el significado del concepto de conversión entre registros de representación (Duval, 1999). Es decir, los estudiantes convierten un problema representado en registro geométrico en unas condiciones representadas en registro algebraico. De la misma forma, cuando los estudiantes generan el discurso para la resolución del problema planteado mediante registro algebraico (resuelven las ecuaciones planteadas) están realizando un tratamiento dentro del mismo registro algebraico.

Por tanto, la extensión del modelo teórico RC consiste, en primer lugar en la ampliación del significado que damos a la expresión afirmación matemática, considerando como tal a cada uno de los datos numéricos o literales en los problemas empíricos. En segundo lugar, RC extendido considera el uso del registro algebraico en la solución de los problemas, en los dos procesos señalados: uso del registro algebraico como proceso de conversión entre registros distintos, y uso del registro algebraico como proceso de tratamiento, dentro de un registro, para resolver sistemas de ecuaciones.

Con estas ampliaciones, es posible identificar los tres tipos de desenlaces: truncamiento y conjetura sin demostración, cuando el proceso desemboca en solución al problema planteado; bucle, cuando el alumno vuelve a la situación de partida una o varias veces, entrando en una trayectoria de bloqueo. Para evidenciar la necesidad de la ampliación del modelo RC, realizamos el análisis de la respuesta de una alumna a un problema empírico (Figura 4). Indicamos los momentos del proceso de resolución en los que son necesarias las ampliaciones de los elementos teóricos del modelo configural. 
Problema 3. Dos círculos son tangentes interiores como se muestra en la figura. Calcula los radios de ambos círculos.

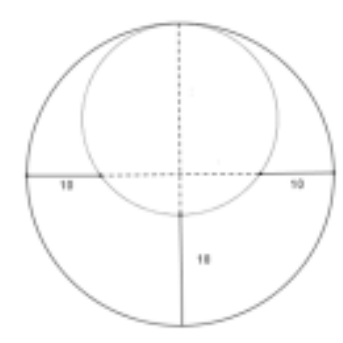

Figura 4. Problema empírico 3

Este problema muestra una situación geométrica particular ("como se muestra en la figura") de dos circunferencias tangentes interiores y pide la medida de un elemento geométrico de dicha situación ("el radio de cada círculo"). Se añaden las medidas de tres segmentos particulares como datos. Por tanto, no se hace mención a propiedades geométricas genéricas y se solicita el cálculo de las medidas de dos segmentos. Se presenta en la Figura 5 la transcripción de la respuesta de la alumna 1:
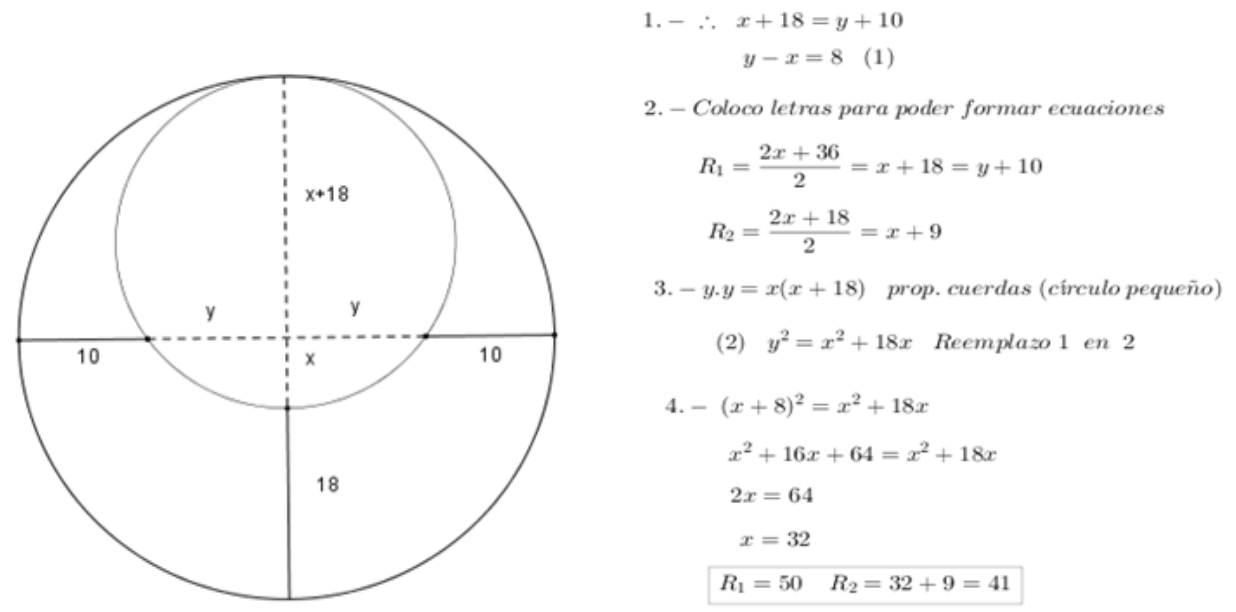

Figura 5. Transcripción de la respuesta de la alumna 1 al problema 3

\section{Análisis de la respuesta desde el modelo $R C$}

La alumna, en 1, plantea una igualdad entre dos expresiones de la medida del radio del círculo mayor según las incógnitas que usa en la configuración inicial (coloco letras para poder formar ecuaciones). El símbolo $\therefore$ se lee "por tanto". Seguidamente, en 2, llama $R 1$ al radio del círculo mayor y $R 2$ al radio del círculo menor que expresa en función de $x$, calculándolos a partir de la definición de radio como mitad del diámetro (aunque no lo cita). Hasta este momento, la alumna está identificando elementos geométricos de ambos círculos (los radios de ambos) y trata de asociarles su medida. El hecho de identificar elementos geométricos se puede interpretar desde RC como realización de aprehensiones operativas puesto que prescinde del resto de la configuración inicial. Sin embargo la asociación de una medida a un elemento geométrico no equivale a la asociación con una afirmación matemática, es decir: no es lo mismo que asociar un teorema, un axioma, una definición, una propiedad. El modelo RC que usamos para realizar el análisis de las soluciones a los problemas clásicos de probar en Geometría no contempla esta acción cognitiva de asociar una medida a un elemento geométrico. En 3, la alumna usa una propiedad de las cuerdas junto a una ecuación, sin relación directa con lo anterior, que resuelve el problema. Esta propiedad 
está vinculada a la identificación de la sub-configuración (parte de la configuración inicial) de la Figura 6:

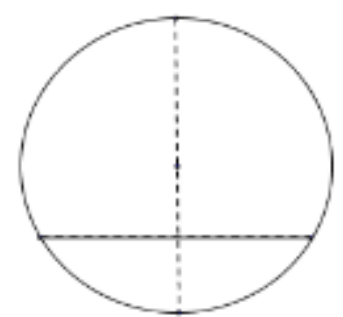

Figura 6. Subconfiguración relevante identificada

Esta identificación constituye una AO, para asociar a la subconfiguración de la Figura 5 la propiedad de las cuerdas: "Si P es un punto en el plano y se fija una circunferencia con centro $O$, entonces para toda recta que pase por $\mathrm{P}$ y corte a la circunferencia en dos puntos $A, B$, se cumplirá que $P A x P B$ es constante". El valor de dicha constante se denomina potencia del punto $P$ respecto de la circunferencia de centro $O$. La asociación entre subconfiguración y afirmación matemática es una $\mathrm{AD}$ que permite realizar una conversión entre registro gráfico y algebraico (propiedad de las cuerdas en forma de relación algebraica). En 4, la alumna realiza un tratamiento dentro del registro algebraico (resuelve las ecuaciones) y da la solución al problema (acción no contemplada en el modelo RC). El desenlace de RC es la solución al problema propuesto. En 3, la alumna realiza un truncamiento; cuando escribe la expresión algebraica realizando la conversión de la propiedad de las cuerdas ya conoce el camino para la resolución al problema ( $\mathrm{RC}$ desemboca en truncamiento). Solo tenía que resolver las ecuaciones planteadas.

En el relato del análisis se mezclan conceptos en el modelo $\mathrm{RC}(\mathrm{AO}$ y $\mathrm{AD}$, coordinación entre ambas, cambios de anclaje) con la aparición de ecuaciones (en registro algebraico), algunas vinculadas a afirmaciones matemáticas genéricas (propiedad de las cuerdas) y otras a la configuración que acompaña el enunciado del problema (medidas 10 y 18 de la configuración inicial, $x+18=y+10$ ). El rol desempeñado por ambos tipos de ecuación en el proceso de resolución del problema es distinto. Las ecuaciones no vinculadas a afirmaciones matemáticas genéricas no son contempladas en el modelo RC.

\section{Análisis con el modelo RC extendido}

\subsection{Ejemplo alumna 2}

La Figura 7 muestra la respuesta de la alumna 2 al problema empírico 3. Se ve una solución que usa afirmaciones geométricas (teorema de Pitágoras) y su conversión en registro algebraico y complementa con relaciones algebraicas (ecuaciones) entre las medidas de los diámetros y radios de ambas circunferencias. Una vez establecidas las ecuaciones resuelve el sistema y logra la solución. 


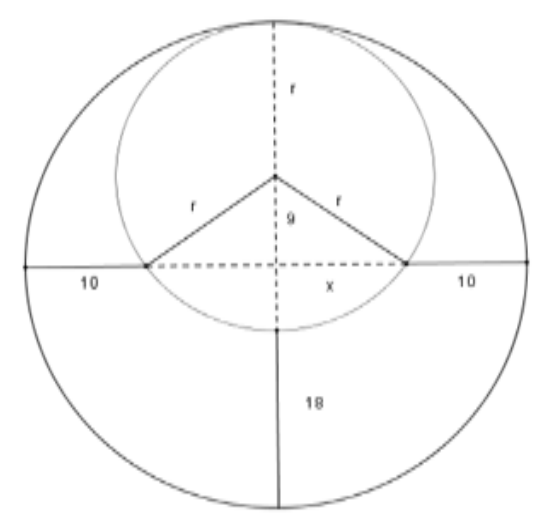

$$
\begin{array}{ll}
\text { 1. }- & 2 r+18=2 R \\
& r+9=R \\
\text { 2. }- & x^{2}+9^{2}=r^{2} \\
\text { 3. }- & x+10=R \\
& x=R-10 \\
& 3 .- \text { en } 2 .-x \text { en } x \\
\text { 4. }- & (R-10)^{2}+81=r^{2} \\
& \text { 1. }- \text { en } 4 .-R \text { en } R \\
(r+9-10)^{2}+81=r^{2} \\
r^{2}-2 r+1+81=r^{2} \\
82=2 r \\
r=41 \\
R=50
\end{array}
$$

Figura 7. Transcripción de la respuesta de la alumna 2 al problema 3

\section{Análisis de la respuesta desde el modelo $R C$ extendido}

1- Identifica los diámetros de ambas circunferencias realizando una AO y les asocia sus medidas $(2 r$ y $2 R)$ mediante una AD.

2- Relaciona ambos segmentos según los datos del problema y expresa la relación en registro algebraico, realizando una conversión pasando del registro gráfico al registro algebraico, $2 r+18=2 R$. Posteriormente simplifica la relación mediante tratamiento obteniendo $r+9=R$

3- Identifica el triángulo (AO) cuyos lados miden $x, 9, r$ (AD). Identifica el triángulo (AO), asumiendo sin demostración que es rectángulo, y le asocia el teorema de Pitágoras $(\mathrm{AD})$. Expresa la relación dada por el teorema de Pitágoras en registro algebraico.

4- Asocia dos expresiones literales $x+10$ y $R$ (AD) al segmento identificado, el radio mayor (AO). Expresa la identidad en registro algebraico y realiza un tratamiento. Indica el paso siguiente: 3 en 2 en la continuación del tratamiento.

5- Sustituye y resuelve el problema.

El proceso de resolución desemboca en una solución de conjetura sin demostración al aceptar sin demostrar que el triángulo identificado es rectángulo. El uso del modelo $\mathrm{RC}$ extendido da cuenta de las coordinaciones cognitivas entre registro geométrico y algebraico al incorporar las ideas del tratamiento y conversión entre registros.

\subsection{Ejemplo alumno 3}


La Figura 8 muestra la respuesta del alumno 3 al problema 3, siendo en este caso una solución totalmente expresada en registro algebraico. Aquí el alumno parte de la definición de la tangente trigonométrica de un ángulo y plantea varias expresiones para un mismo ángulo. Realiza el tratamiento adecuado en el registro algebraico y resuelve.
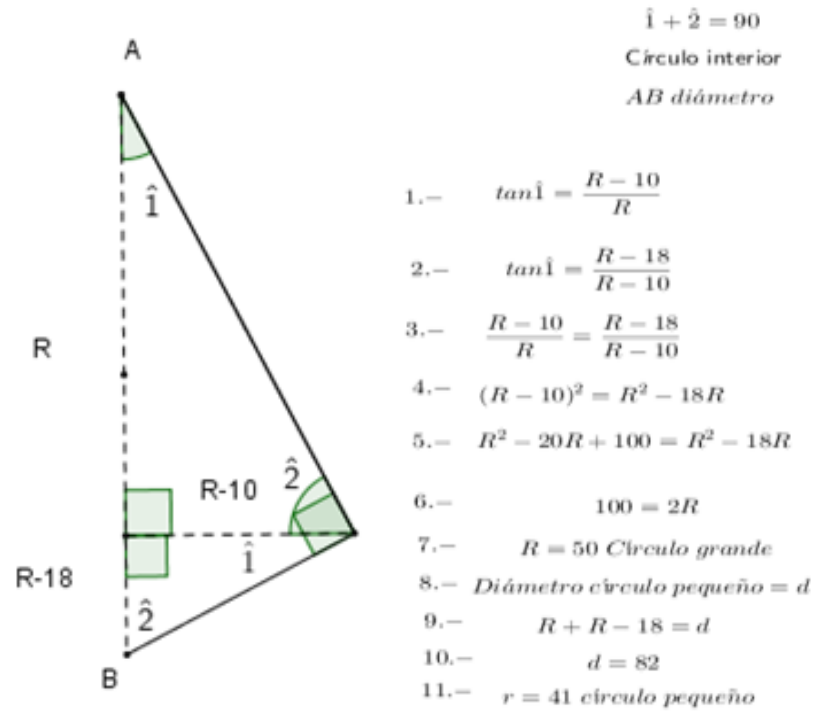

Figura 8. Transcripción de la respuesta del alumno 3 al problema 3

\section{Análisis de la respuesta desde el modelo $R C$ extendido}

1. En la figura del enunciado, el alumno identifica tres triángulos rectángulos con ángulos rectos marcados en color (AO). Marca los ángulos agudos de cada triángulo en $\wedge^{\wedge} 1 \mathrm{y}^{\wedge} 2$. Estas acciones se corresponden a AO (identificación de subconfiguraciones) y $\mathrm{AD}$ al asociarles sus valores ${ }^{\wedge} 1, \wedge 2$.

2. También realiza una $A D$ cuando asocia el valor de 90 a la medida del ángulo ${ }^{\wedge} 1+^{\wedge} 2$. Igualmente, realiza una $\mathrm{AO}$ cuando identifica un ángulo inscrito que abarca una semicircunferencia y le asocia su medida $90^{\circ}$.

3. Identifica el radio del círculo grande (AO) y le asocia su valor $R(\mathrm{AD})$.

El alumno no ha demostrado que los diámetros se cortan formando ángulos rectos. Por ese motivo, al igual que en el ejemplo anterior, se considera que hay un desenlace del RC de conjetura sin demostración. Identifica segmentos que forman parte del radio del círculo grande y le asocia sus medidas, de acuerdo con los datos del problema $(R$, $R-10$ y $R-18)$. Todas estas identificaciones y asociaciones son acciones coordinadas entre AO (al aislar parte de la subconfiguración y expresarla en registro algebraico) y $\mathrm{AD}$ (al asociar las medidas). En 1 y 2, aparece la expresión de la tangente del ángulo $\wedge 1$ en forma de dos cocientes, a partir de identificar la subconfiguración de la Figura 9.

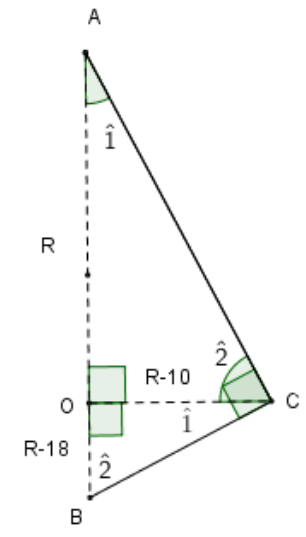


Figura 9. Subconfiguración relevante del problema 3 para el alumno 3

A partir de la identificación de las subconfiguraciones: triángulos $A O C$ y $C O B$ (lo que constituyen dos $\mathrm{AO}$ ) el alumno asocia la definición de la tangente trigonométrica del mismo ángulo en dos triángulos distintos (lo que constituye en cada asociación una $\mathrm{AD})$. En el momento en que el alumno identifica el mismo ángulo en ambos triángulos se produce truncamiento (aunque cabe considerar este desenlace como conjetura sin demostración por aceptar, sin demostrarlo, que los diámetros son perpendiculares). El alumno ya sabe cómo resolver el problema. A partir del punto 3 y hasta el final, mediante el tratamiento adecuado dentro del registro algebraico (resolviendo el sistema de ecuaciones), el alumno encuentra la solución. Esta solución utiliza solamente registro algebraico en su presentación en lenguaje escrito. Ni siquiera ha dado la definición de la tangente trigonométrica de un ángulo como "cateto opuesto partido por cateto adyacente". Se ha limitado a representar la situación particular del problema en lenguaje algebraico para hallar las relaciones (ecuaciones) que necesitaba para llegar a la solución.

\section{Discusión y conclusiones}

Con este trabajo se indica la pertinencia de ampliar el modelo RC, usado en el análisis de la resolución de problemas de probar de geometria clásicos, en un contexto de lápiz y papel, cuando se resuelven problemas empíricos. Se han analizado tres soluciones a un problema empírico, correspondientes a tres estrategias de resolución. Una en la que la alumna 1 utiliza una propiedad geométrica general (propiedad de las cuerdas), que convierte al registro algebraico y que complementa con una relación algebraica, a partir de la situación particular de la figura (igualdad entre dos expresiones del radio mayor) para hallar la solución. Esta solución ha servido para introducir la necesidad del modelo RC extendido. La resolución se podría considerar que constituye un razonamiento geométrico general en registro algebraico. En la segunda resolución, la alumna 2 busca una relación entre los radios de ambas circunferencias, que le lleva a establecer una ecuación (que expresa la relación entre radios) y que complementa con una propiedad genérica (teorema de Pitágoras). En esta solución hay una mezcla de razonamientos y registros algebraico y geométrico. En la tercera, el alumno 3 expresa de dos formas la tangente trigonométrica del mismo ángulo, que no define, obteniendo una ecuación con una incógnita que resuelve el problema, con una solución en registro algebraico.

Los ejemplos descritos muestran el potencial explicatorio del modelo RC extendido, cuando se usa para el análisis de la resolución de los problemas empíricos. Con el modelo extendido podemos analizar las respuestas de los alumnos a problemas empíricos y así avanzar en el estudio de características del razonamiento de los alumnos cuando resuelven problemas de probar y calcular en contexto geométrico, con lápiz y papel, tanto si usan un registro solo geométrico como si introducen en su discurso el registro algebraico.

Todas las respuestas analizadas suponen cierta la perpendicularidad entre los diámetros de la circunferencia mayor pues la utilizan, aunque sea implícitamente, para resolver el problema, pero no la explicitan. Este es un problema recurrente en geometría. A veces utilizamos enunciados con figuras "mudas" (sin ninguna marca) y decimos simplemente, como en nuestro problema: Dos círculos son tangentes interiores como se muestra en la figura. Esta forma de proceder no es coherente con 
la exigencia a los alumnos de que no deben basar sus conjeturas en lo que parece que se cumple en la configuración inicial que acompaña a los enunciados. Esta reflexión implica que deberíamos restringir el uso de las figuras mudas en el enunciado de los problemas. Tal vez, el hecho de no incluir la hipótesis de la perpendicularidad de los diámetros del círculo mayor ha propiciado la aparición de varias soluciones, evidenciando que es muy complicado estudiar los procesos desarrollados por los alumnos durante la resolución de un problema a través de un contenido fijado a priori, como por ejemplo "conocimiento susceptible de ser utilizado" (Clemente \& Llinares, 2015).

Con los problemas empíricos ampliamos los objetivos de nuestra investigación. Desde el registro geométrico, que necesita conocimiento de afirmaciones matemáticas y capacidad de coordinación para relacionarlas y generar luego un discurso expresado según las normas del razonamiento deductivo, pasamos a considerar estrategias de resolución que están expresadas en una combinación de registros geométricosalgebraicos. Una característica de los problemas empíricos es el registro algebraico, que es un lenguaje potente. Aunque precisa conocimiento matemático igual que el registro geométrico, el rol desempeñado por la coordinación entre hechos geométricos pierde relevancia. Cuando el alumno es capaz de expresar (representar) la situación geométrica del problema en forma de ecuaciones (registro algebraico), estas se resuelven de manera "algorítmica", simplificándose la tarea de elaborar un discurso deductivo al responder a la característica del propio problema ("Calcular"). Se precisan investigaciones de largo periodo de observación para evidenciar analogías/diferencias, si las hay, entre el razonamiento de los alumnos en diferentes tipos de problemas por causa de la formación recibida, según haya predominado el registro geométrico o el algebraico.

La solución del problema que hemos presentado se desencadena a partir de la identificación de una subconfiguración relevante, aunque el desencadenante no funcione desde el anclaje visual al discursivo (Clemente, Llinares \& Torregrosa, 2017). Hay evidencias que sugieren que el conocimiento teórico de los alumnos les hace "buscar" la subconfiguración que se ajuste a dicho conocimiento teórico (Llinares \& Clemente, 2014). Sin embargo, son necesarios más trabajos de investigación para explicar el cambio de anclaje (Duval, 1998). La identificación de características que explicasen cómo realizan los alumnos los cambios de anclaje tendría implicaciones en la enseñanza de las matemáticas en general y de la geometría en particular: identificar cómo los estudiantes realizan la asociación entre lo que identifica en una configuración inicial y las afirmaciones matemáticas que conoce. En otras palabras: por qué hay estudiantes que resuelven los problemas, generalmente, con cambio de anclaje de visual a discursivo y otros que realizan la asociación en sentido contrario: de discursivo a visual.

\section{Referencias}

Balacheff, N. (2008). The role of the researcher's epistemology in mathematics education: an essay on the case of proof. ZDM, 40, 501-512.

Clemente, F., \& Llinares, S. (2015). Formas del discurso y razonamiento configural de estudiantes para maestro en la resolución de problemas de Geometría. Enseñanza de las Ciencias, 33(1), 9-27. 
Clemente, F., Llinares, S., \& Torregrosa, G. (2017). Visualización y razonamiento configural. BOLEMA, 31(57), 497-516.

Duval, R. (1995). Geometrical Pictures: kinds of representation and specific processing. En R. Sutherland \& J. Mason (Eds.), Exploiting Mental Imagery with Computers in Mathematics Education (pp. 142-157). Berlín: Springer.

Duval, R. (1998). Geometry from a cognitive Point of view. En C. Mammana \& V. Villani (Eds.), Perspectives on the Teaching of Geometry for the 21st Century. An ICMI Study (pp. 37-52). Dordrecht: Kluwer.

Duval, R. (1999). Semiosis y pensamiento humano. Cali, Colombia: Univalle.

Fiallo, J., \& Gutiérrez, A. (2017). Analysis of the cognitive unity or rupture between conjecture and proof when learning to prove on a grade 10 trigonometry course. Educational Studies in Mathematics, 96(2), 145-167.

Hanna, G., \& Jahnke, N. (1996). Proof and proving. En A. J. Bishop, K. Clements, C. Keitel, J. Kilpatrick \& C. Laborde (Eds.), International Handbook of mathematics education (pp. 877-908). Dordrecht: Kluwer.

Harel, G., \& Sowder, L. (1998). Students' proof schemes: results from exploratory studies. En E. Dubinsky, A. Schoenfeld \& J. Kaput (Eds.), Research on Collegiate Mathematics Education III (pp. 234-283). Providence: AMS.

Hilbert, T., Renkl, A., Kessler, S., \& Reiss, K. (2008). Learning to prove in geometry: learning from heuristic examples and how it can be supported. Learning and Instruction, 18, 54-65.

Ibañes, M. (2002). Cuatro cuestiones en torno al aprendizaje de la demostración. En M. F. Moreno, F. Gil, M. Socas, J. D. Godino (Eds.), Investigación en Educación Matemática $V$ (pp. 11-26). Almería: Universidad de Almería.

Ibañes, M., \& Ortega, T. (2005). Dimensiones de la demostración matemática en bachillerato. Números, 61, 19-40.

Komatsu, K. (2016). A framework for proofs and refutations in school mathematics: Increasing content by deductive guessing. Educational Studies in Mathematics, 92(2), 147-162.

Llinares, S., \& Clemente, F. (2014). Characteristics of pre-service primary school teachers' configural reasoning. Mathematical Thinking and Learning, 16(3), 234250.

Miyazaki, M., Fujita, T., \& Jones, K. (2017). Students' understanding of the structure of deductive proof. Educational Studies in Mathematics, 94(2), 223-239

Prior, J. y Torregrosa, G. (2013). Razonamiento Configural y procedimientos de verificación en contexto geométrico. Revista Latinoamericana de Investigación en Matemática Educativa, 16(3), 339-368.

Reiss, K.M., Heinze, A., Renkl, A., \& Groß, C. (2008). Reasoning and proof in geometry: Effects of a learning environment based on heuristic worked-out examples. $Z D M, 40(3), 455-467$.

Robotti, E. (2012). Natural language as a tool for analyzing the proving process: the case of plane geometry proof. Educational Studies in Mathematics, 80(3), 433-450. 
Torregrosa, G. (2013). Razonamiento configural y enseñanza de la geometría. UNO. Revista de Didáctica de la Matemática, 62, 49-56.

Torregrosa, G., \& Quesada, H. (2007). Coordinación de procesos cognitivos en Geometría. Revista Latinoamericana de Investigación en Matemática Educativa,10(2), 275-300.

Torregrosa, G.; Quesada, H., \& Penalva, M.C. (2010). Razonamiento configural como coordinación de procesos Visualización. Enseñanza de las Ciencias,28(3), 327340.

Zazkis, R., \& Dubinsky, E. (1996). Dihedral groups: a tale of two interpretations. CBMS. Issues in Mathematics Education, 6, 61-82.

Zazkis, R., Dubinsky, E., \& Dautermann, J. (1996). Coordinating visual and analytical estrategies. A Study of students' understanding of the group $\mathrm{D}_{4}$. Journal for Research in Mathematics Education, 27(4), 435-457.

\section{Referencia al autor}

Germán Torregrosa, Universidad de Alicante (España) german.torregrosa@ua.es 


\title{
Coordination of cognitive processes in problem solving: relationship between geometry and algebra.
}

\author{
Germán Torregrosa, Universidad de Alicante (Spain)
}

The study of the characteristics of students' reasoning when they solve problems of geometry with pencil and paper has led us to elaborate a theoretical model called Configural Reasoning (CR). CR implies that students develop a coordinated action between Operative and Discursive Apprehensions, associating mathematical affirmations and/or making modifications in the initial configuration when they are solving a geometry problem with pencil and paper.

From a cognitive standpoint, the CR model explains the development of the process followed by students when facing geometrical problems of proof. Nonetheless, the model does not well cover the analysis of students' answers in the resolution of empirical geometrical problems; that is, when students solve problems in which the initial conditions are not geometric properties but concrete measures (numerical or literal). Due to the dominance of empirical geometrical problems in secondary education, the greater relation of these problems with students' daily life and the greater motivation involved, it is necessary to investigate new approaches to the CR model in order to expand the set of problems that can be analyzed.

Extended Configural Reasoning (ECR) is the result of research around such new approach. This paper presents the extension of the CR model for the analysis of the resolution of empirical problems of geometry, in which the initial data are numerical or literal. The extension of the CR model consists of considering that in Discursive Apprehensions, within the meaning of "mathematical affirmation" including theorems, axioms, definitions, properties as well as the initial conditions (hypotheses) of empirical problems, expressed as numerical or literal data. Furthermore, this new model considers the algebraic register generated in the discourse during the resolution of a problem, within a geometrical context. This inclusion of the algebraic register in the model draws on the concepts of conversion and treatment of the Theory of Semiotic Systems by Duval. After the analysis of several resolutions to an empirical problem with the ECR model, newer aspects have come to explain the "traditional" behavior of students in their learning of Geometry. For future research, we could consider the use of "silent" figures. We could also change our focus to incorporate the analysis of resolution strategies expressed through hybrid geometrical-algebraic registers. 